\title{
Broad Beam-Induced Fragmentation and Joining of Tungsten Oxide Nanorods: Implications for Nanodevice Fabrication and the Development of Fusion Reactors
}

\author{
Manoj K Rajbhar ${ }^{1}$, Wolfhard Möller ${ }^{2}$, Biswarup Satpati ${ }^{3}$, Unnikrishnan Manju ${ }^{4}$, \\ Yatendra S Chaudhary ${ }^{4}$, Shyamal Chatterjee*1 \\ ${ }^{1}$ School of Basic Sciences, Indian Institute of Technology Bhubaneswar, Bhubaneswar \\ 751007, India \\ ${ }^{2}$ Institute of Ion Beam Physics and Materials Research, Helmholtz-Zentrum Dresden- \\ Rossendorf, 01328 Dresden, Germany
}

${ }^{3}$ Surface Physics \& Material Science Division, Saha Institute of Nuclear Physics, Kolkata 700 064, India

${ }^{4}$ Materials Chemistry Department. CSIR-Institute of Minerals and Materials Technology, Bhubaneswar, 751013, India

*Email: Shyamal@iitbbs.ac.in 


\section{Supplementary Information (S1)}
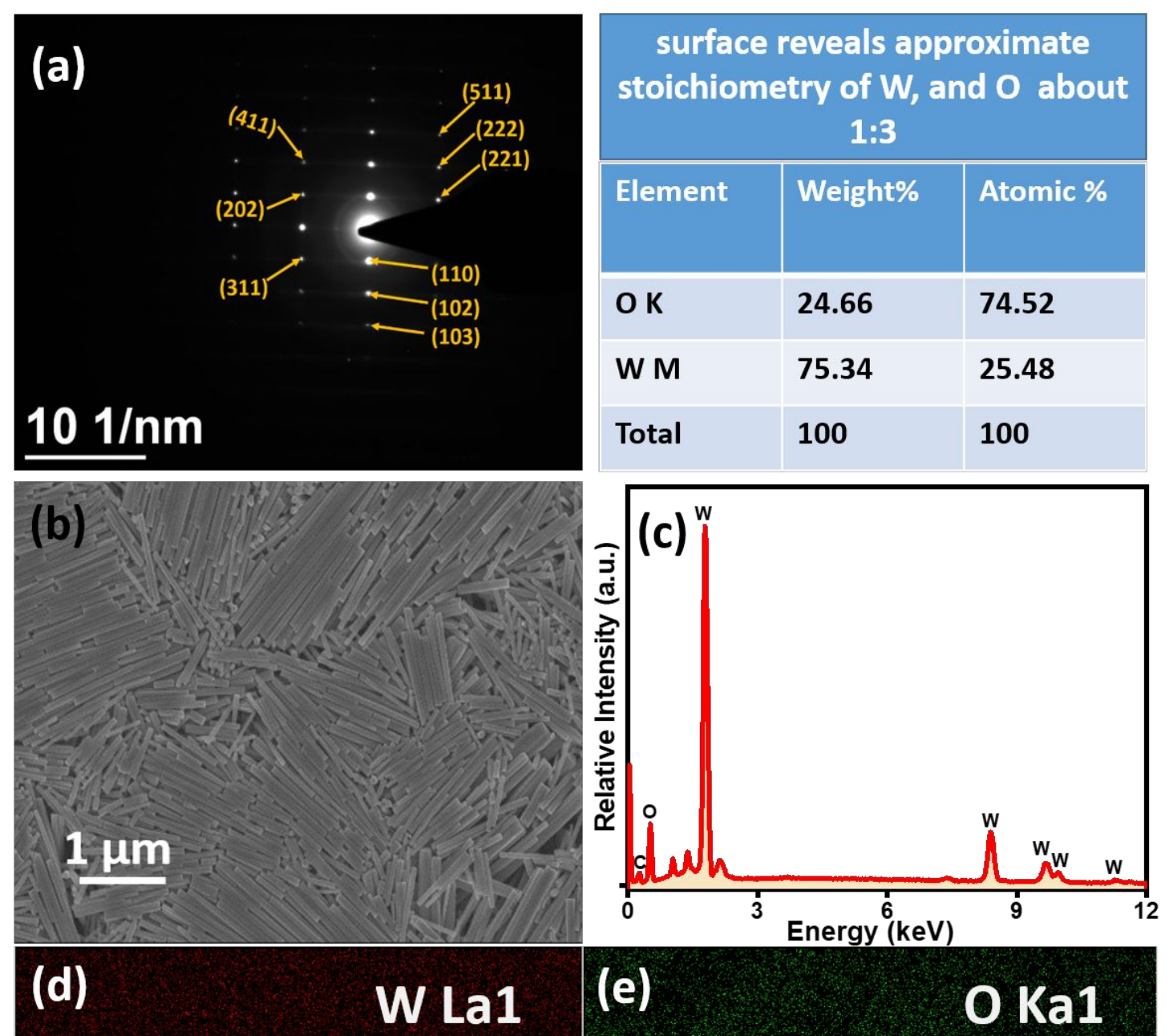

\section{(e)}

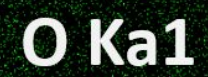

Fig. S1. The selected area (electron) diffractogram of single nanorods showing all possible crystalline planes (a). The EDS spectrum of pristine $\mathrm{WO}_{3}$ nanorods having characteristic peaks from $\mathrm{W}$ and $\mathrm{O}(\mathrm{c})$, over surface area shown in the FESEM image of fig (b), and element mapping image for $\mathrm{W}(\mathrm{d})$, and $\mathrm{O}(\mathrm{e})$. The surface reveals approximate stoichiometry of $\mathrm{W}$, and O of about 1:3. 


\section{Supplementary Information (S2)}

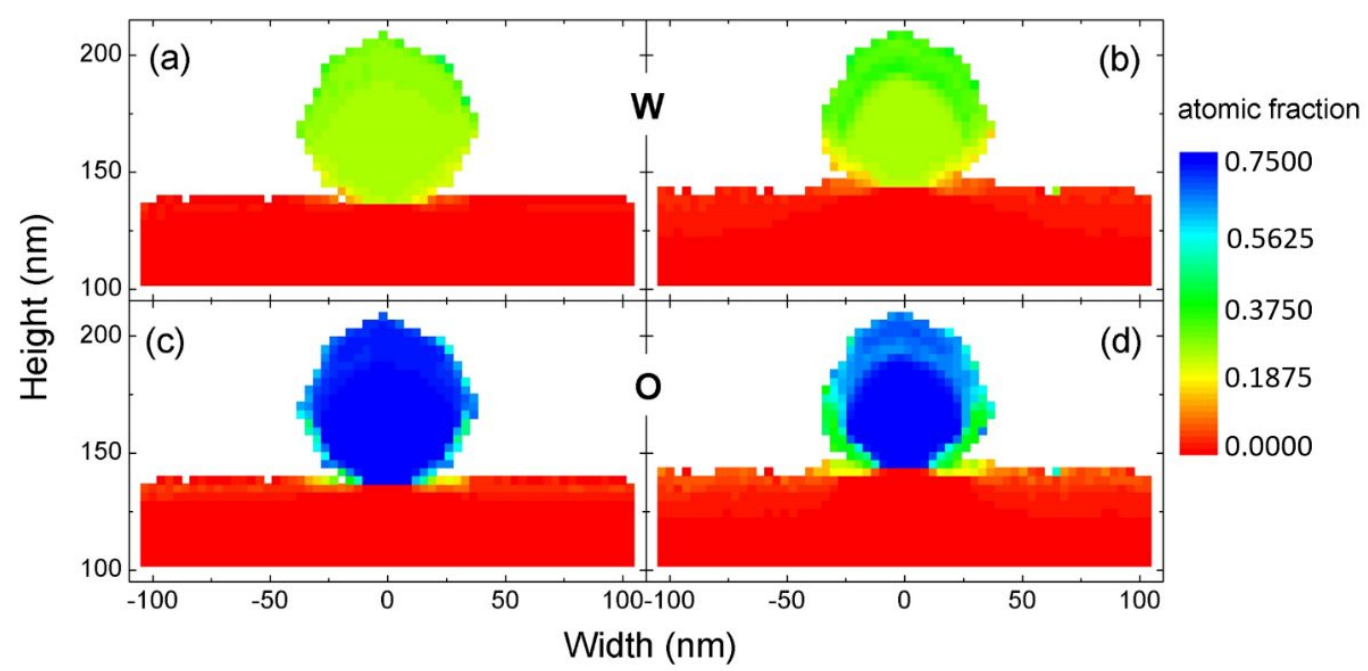

Fig. S2: Cross-sectional view of elemental profiles of $\mathrm{W}(\mathrm{a}, \mathrm{b})$ and $\mathrm{O}(\mathrm{c}, \mathrm{d})$ in an infinitely long $\mathrm{WO}_{3}$ nanowire of $70 \mathrm{~nm}$ diameter on a Si substrate of $210 \mathrm{~nm}$ width after $5 \mathrm{keV} \mathrm{Ar}^{+}$irradiation at fluences of $1 \times 10^{16} \mathrm{~cm}^{-2}(\mathrm{a}, \mathrm{c})$ and $3 \times 10^{16} \mathrm{~cm}^{-2}(\mathrm{~b}, \mathrm{~d})$. The results have been averaged over a central slice of 7 nm thickness. 


\section{Supplementary Information (S3)}
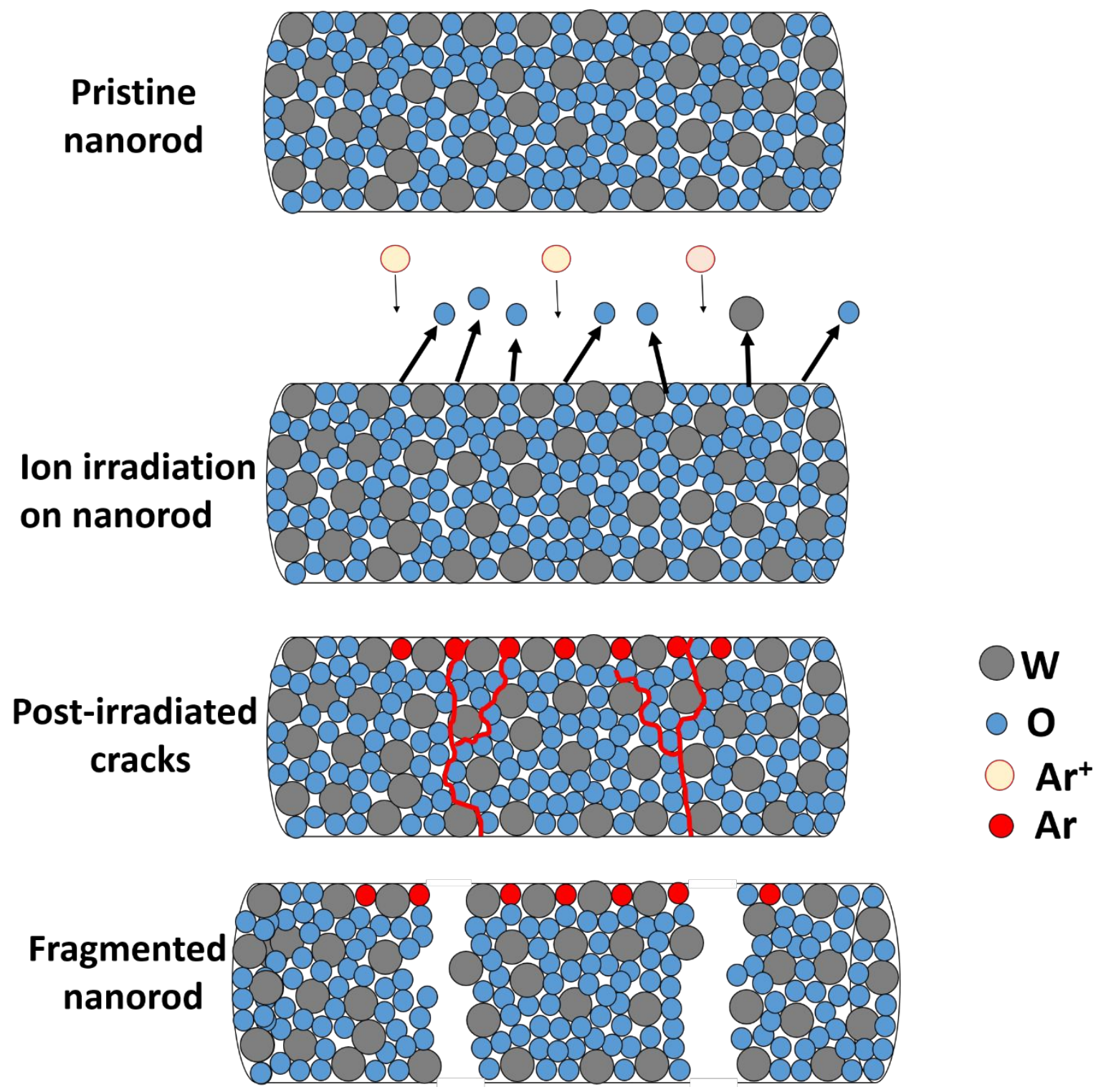

Fig. S3. Schematic of ion beam induced fragmentation of tungsten oxide nanorod. For simplicity of representation, atoms in the nanorod are placed arbitrarily. 


\section{Supplementary Information (S4)}

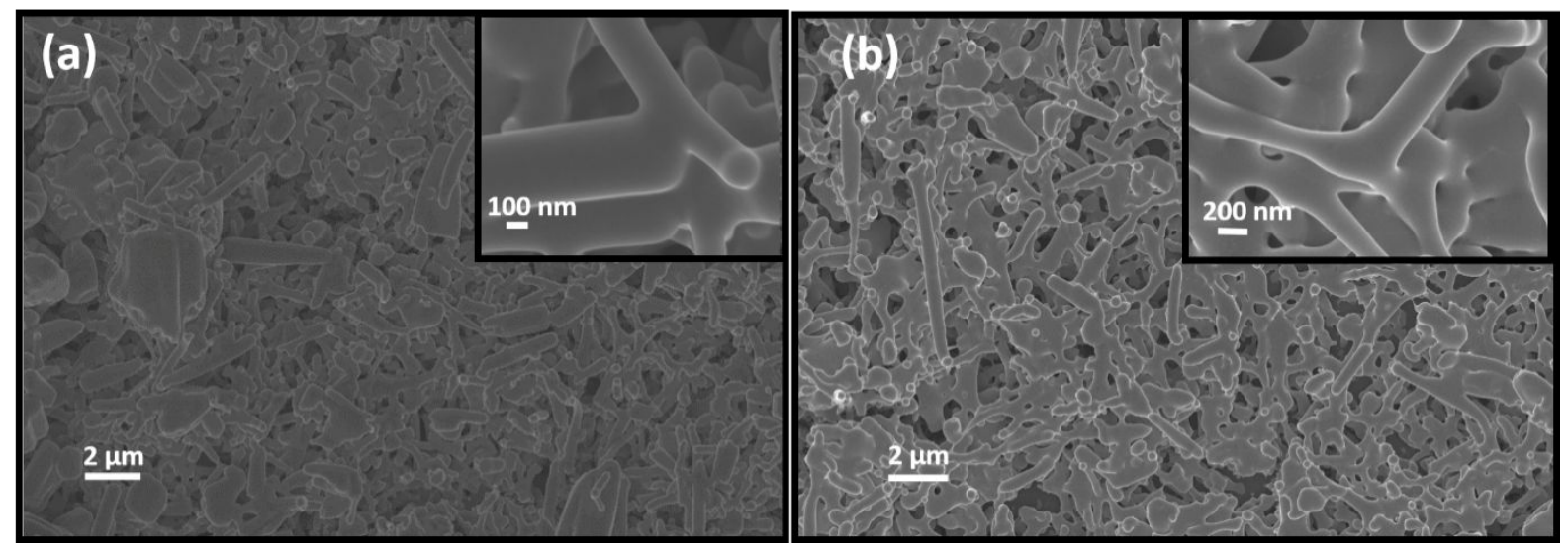

Fig.S4. FESEM images of irradiated tungsten oxide nanorods at $200 \mathrm{keV} \mathrm{Ar}^{+}$at Ion fluences of (a) $1 \times$ $10^{16}$ ions.cm ${ }^{-2}$ and (b) $3 \times 10^{16}$ ions.cm ${ }^{-2}$.

\section{Supplementary Information (S5)}

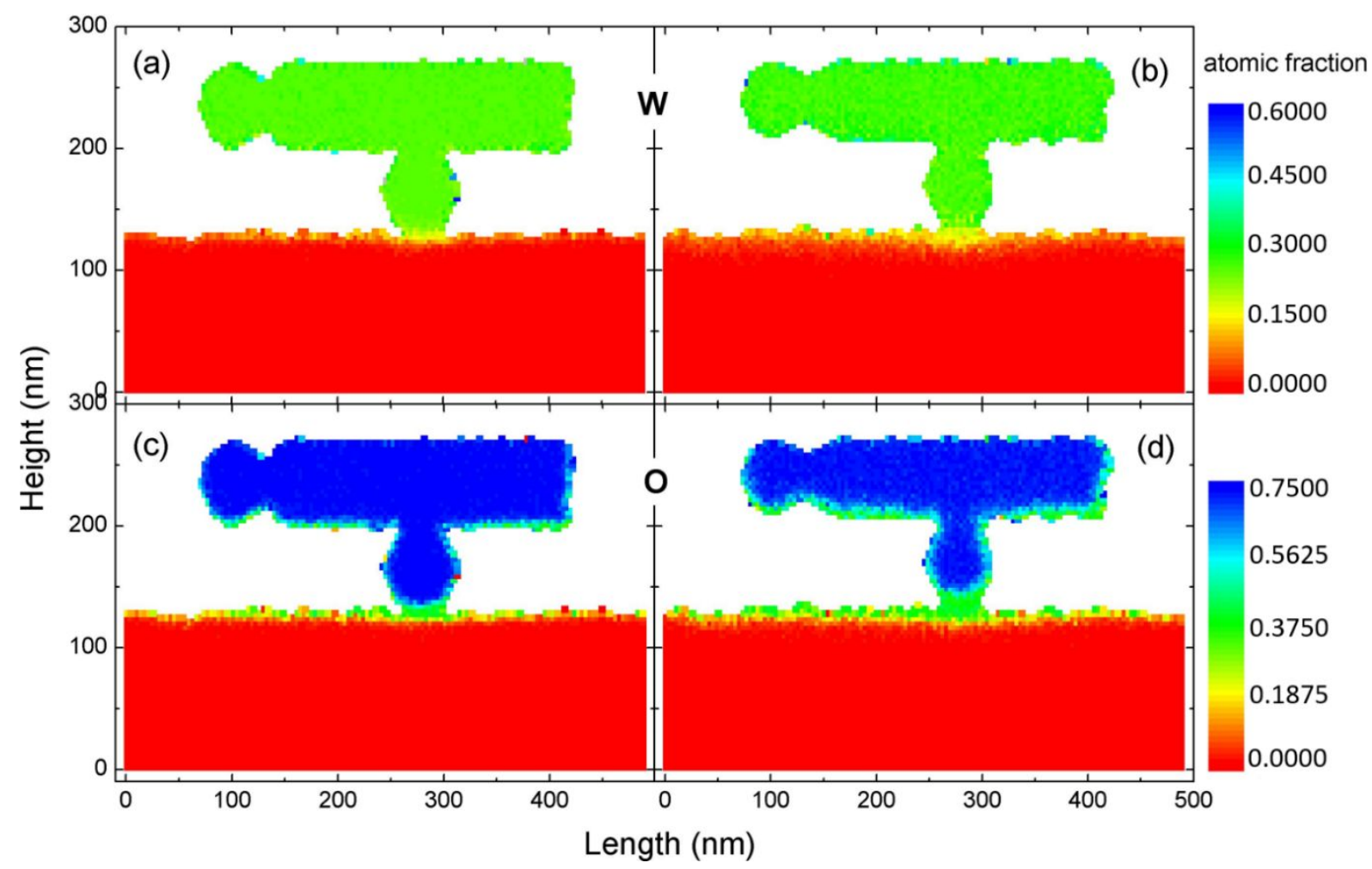

Fig. S5. Elemental profiles of W (a, b) and O (c, d) after $200 \mathrm{keV} \mathrm{Ar}^{+}$irradiation at fluences of 1 $\times 10^{16} \mathrm{~cm}^{-2}(\mathrm{a}, \mathrm{c})$ and $3 \times 10^{16} \mathrm{~cm}^{-2}(\mathrm{~b}, \mathrm{~d})$. The results have been averaged over a central slice of $7 \mathrm{~nm}$ thickness.

\section{Supplementary Information (S6)}




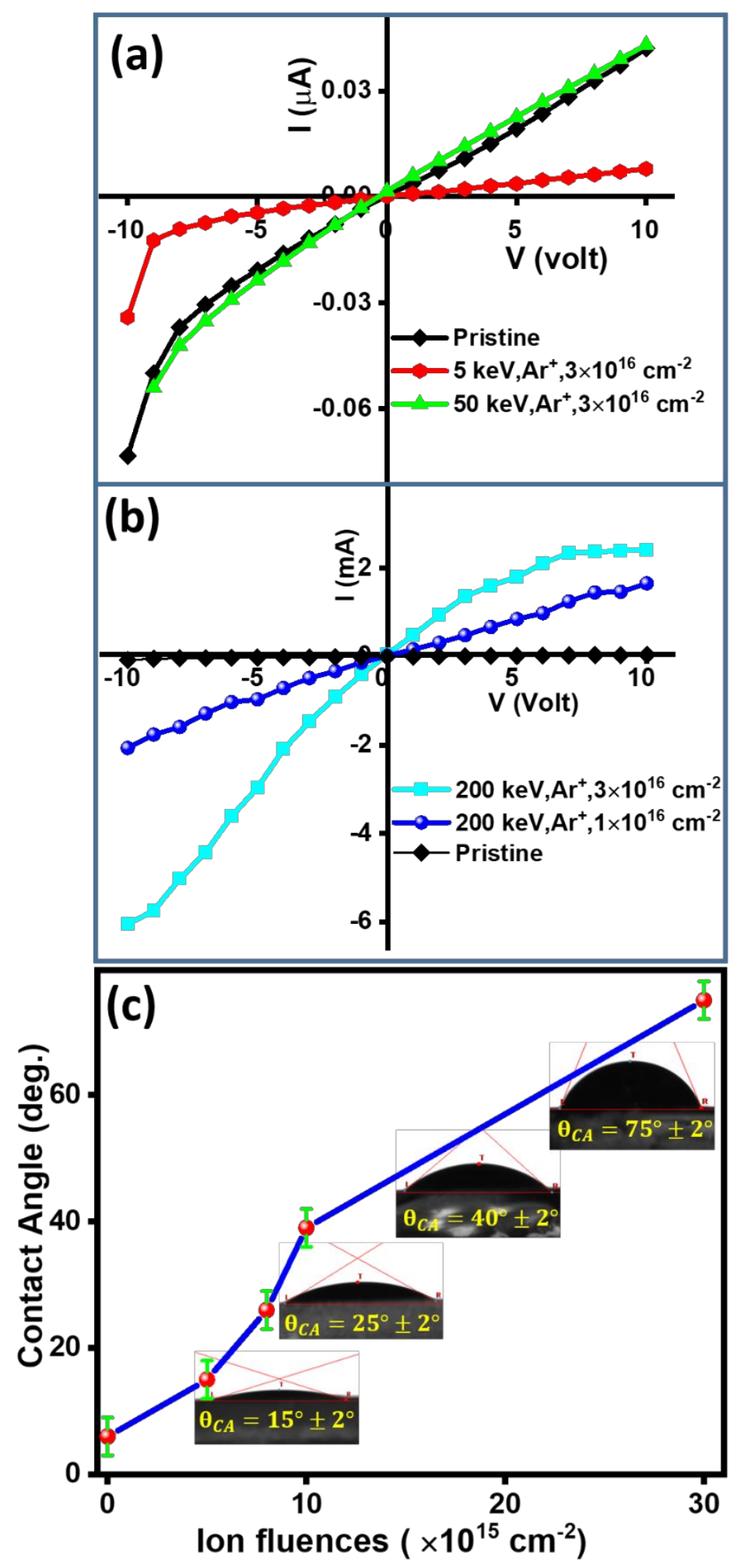

Fig. S6 : I-V characteristics curve of $\mathrm{WO}_{3}$ nanorods at different ion irradiation energies $(5 \mathrm{keV}$, $50 \mathrm{keV}$ ) (a), and $200 \mathrm{keV}$ with varying fluences of $1 \times 10^{16} \mathrm{~cm}^{-2}$, and $3 \times 10^{16} \mathrm{~cm}^{-2}(\mathrm{~b})$. The Contact angle images of water droplets, including the graphical plot of contact angle vs. ions 
fluences of ion beam irradiation Energy $(5 \mathrm{keV})$ on $\mathrm{WO}_{3} \mathrm{NRs}$ arrays of the pristine and irradiated sample (c).

\section{Supplementary Information (S7)}

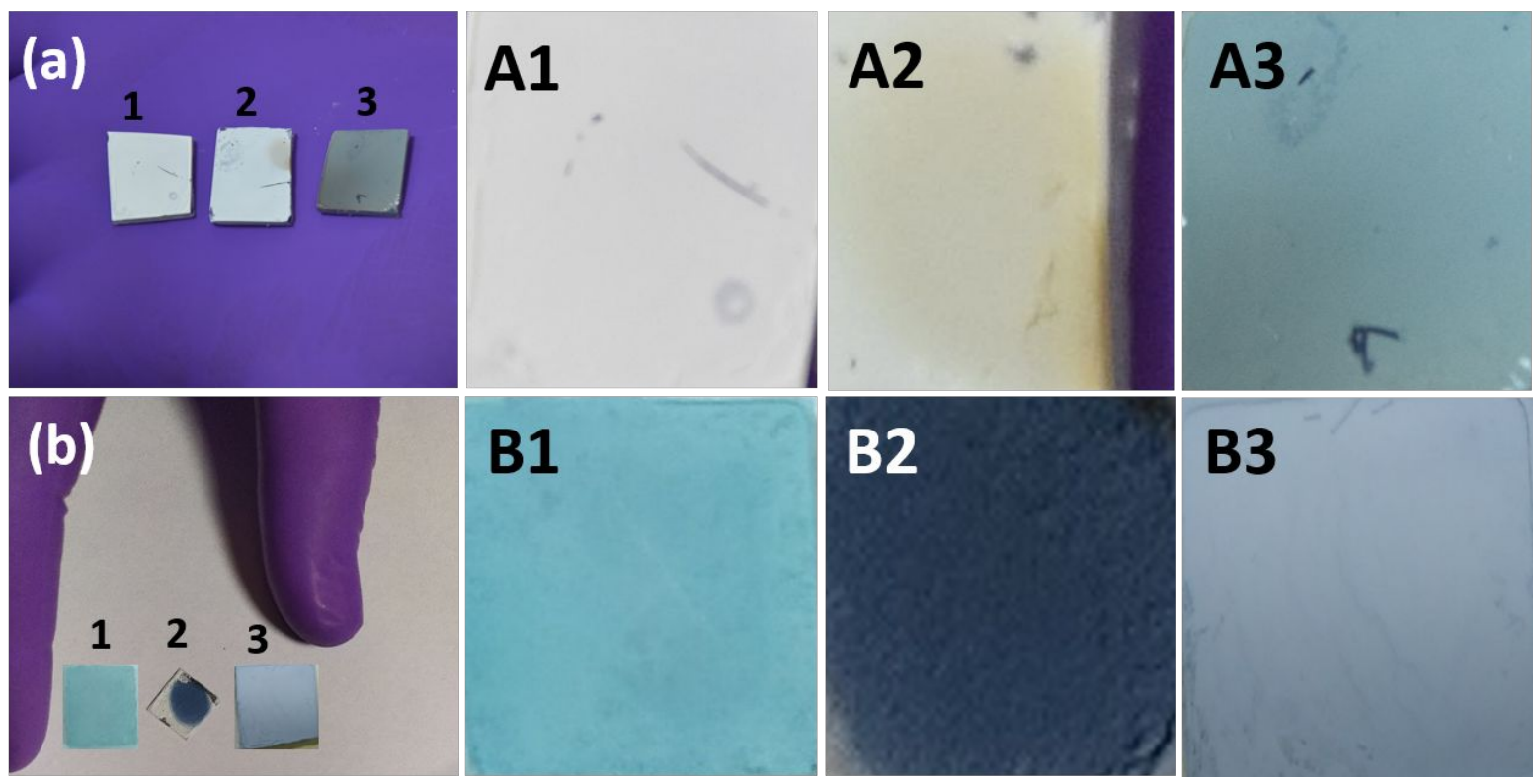

Fig. S7: Photographs of pristine (A1), $5 \mathrm{keV} \mathrm{Na}$ at $1 \times 10^{16} \mathrm{~cm}^{-2}$ irradiated (A2), $5 \mathrm{keV} \mathrm{Ar}^{+}$at $1 \times 10^{16}$ $\mathrm{cm}^{-2}$ irradiated (A3) and all three shown in (a). $5 \mathrm{keV} \mathrm{N} \mathrm{N}^{+}$at $1 \times 10^{16} \mathrm{~cm}^{-2}$ irradiated (B1), $5 \mathrm{keV} \mathrm{Ar}^{+}$at $3 \times 10^{16} \mathrm{~cm}^{-2}$ irradiated (B2), 24 hour UV irradiated (B3) and all three shown in (b). 


\section{Supplementary Information (S8)}
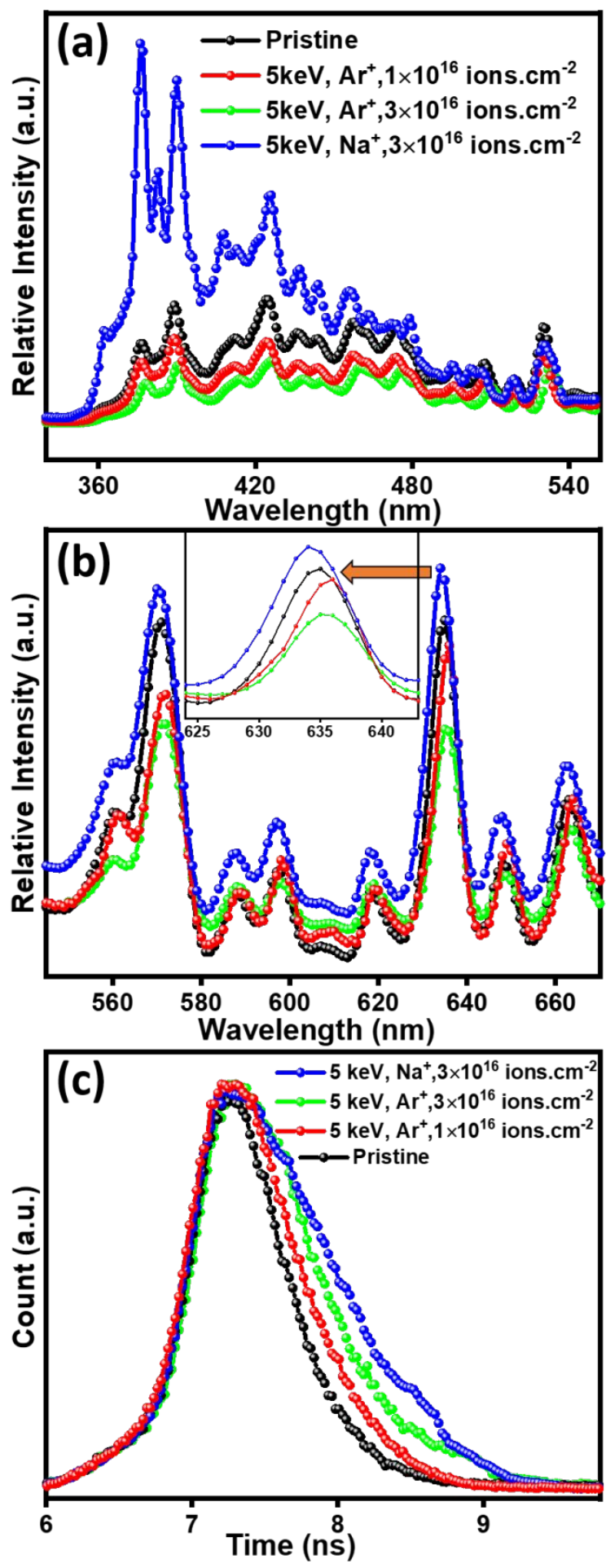

Fig S8: (a) PL spectra of pure $\mathrm{WO}_{3}$ pristine (black), $5 \mathrm{keV} \mathrm{Ar}+$ ion irradiated at fluences $1 \times 10^{16} \mathrm{~cm}^{-2}$ (Red), at fluence $3 \times 10^{16} \mathrm{~cm}^{-2}$ (green), and $5 \mathrm{keV} \mathrm{Na}^{+}$ions irradiated at fluences (Blue) from wavelength range $350 \mathrm{~nm}$ to $550 \mathrm{~nm}$, (a) and from wavelength range from $550 \mathrm{~nm}$ to $650 \mathrm{~nm}$ (b), PL lifetime analysis for rising time and decay time curve of upconversion luminescence (c). 
Supplementary Information (S9)

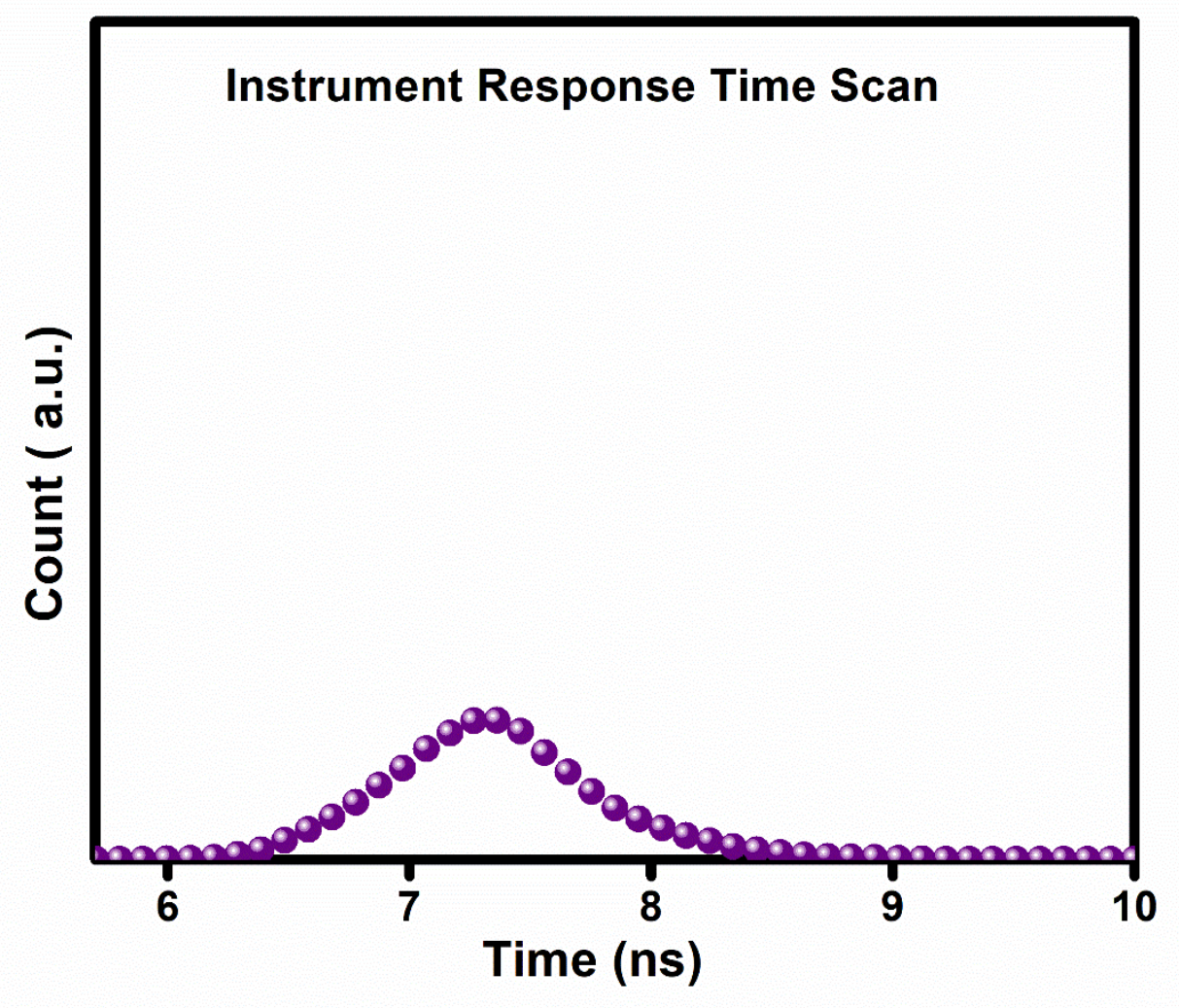

Fig S9. Instrument response time scan of PL study.

\section{Supplementary Information (S10)}

Table S1: Sputtering yields of $\mathrm{W}, \mathrm{O}$ and $\mathrm{Si}$ atoms for different energies and fluence obtained using TRI3DYN simulation

\begin{tabular}{|c|c|c|c|c|}
\hline \multirow{3}{*}{$\begin{array}{c}\text { Energies of } \\
\text { Irradiation }\end{array}$} & $\begin{array}{c}\text { Ion fluences } \\
\left(\mathbf{1} \times \mathbf{1 0}^{\mathbf{1 6}} \mathbf{c m}^{-\mathbf{2}}\right)\end{array}$ & \multicolumn{3}{|c|}{ The sputtering yields } \\
\cline { 2 - 5 } & (Number of sputtered atoms per incident ion) \\
\hline & 0.02 & $\mathbf{W}$ atom & $\mathbf{O}$ atom & Si atom \\
\cline { 2 - 5 } & 0.06 & 0.06498 & 0.8481 & 0.6344 \\
\cline { 2 - 5 } & 0.10 & 0.07309 & 0.8232 & 0.6226 \\
\cline { 2 - 5 } & 0.14 & 0.06478 & 0.8222 & 0.5818 \\
\cline { 2 - 5 } & 0.18 & 0.07254 & 0.7628 & 0.5976 \\
\cline { 2 - 5 } & 0.22 & 0.07930 & 0.7889 & 0.5788 \\
\cline { 2 - 5 } & 0.26 & 0.07347 & 0.7303 & 0.5887 \\
\hline
\end{tabular}




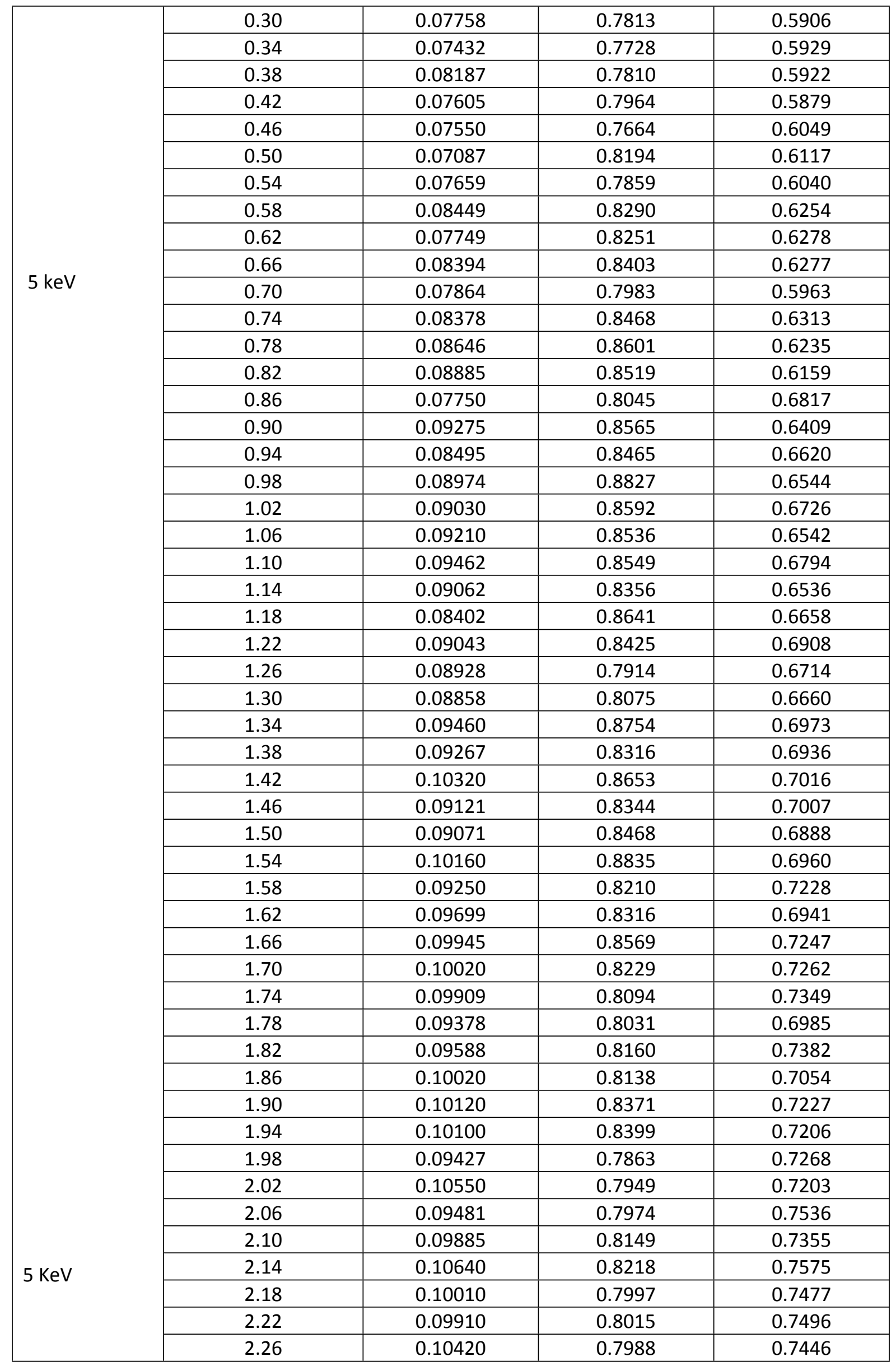




\begin{tabular}{|c|c|c|c|c|}
\hline \multirow{1}{*}{} & 2.30 & 0.10400 & 0.8041 & 0.7369 \\
\cline { 2 - 5 } & 2.34 & 0.09766 & 0.7809 & 0.7836 \\
\cline { 2 - 5 } & 2.38 & 0.10350 & 0.8273 & 0.7326 \\
\cline { 2 - 5 } & 2.42 & 0.11410 & 0.8172 & 0.7520 \\
\cline { 2 - 5 } & 2.46 & 0.10410 & 0.7766 & 0.7301 \\
\hline 2.50 & 0.09859 & 0.8003 & 0.7125 \\
\cline { 2 - 5 } & 2.54 & 0.10920 & 0.7658 & 0.7484 \\
\cline { 2 - 5 } & 2.58 & 0.10770 & 0.7892 & 0.7748 \\
\cline { 2 - 5 } & 2.62 & 0.09874 & 0.7720 & 0.7757 \\
\cline { 2 - 5 } & 2.66 & 0.10550 & 0.7734 & 0.7595 \\
\cline { 2 - 5 } & 2.70 & 0.10340 & 0.7651 & 0.7516 \\
\cline { 2 - 5 } & 2.74 & 0.11500 & 0.7924 & 0.7661 \\
\cline { 2 - 5 } & 2.78 & 0.11090 & 0.7771 & 0.7749 \\
\cline { 2 - 5 } & 2.82 & 0.10690 & 0.7989 & 0.8023 \\
\cline { 2 - 5 } & 2.86 & 0.10810 & 0.8157 & 0.7799 \\
\cline { 2 - 5 } & 2.90 & 0.10400 & 0.7935 & 0.7837 \\
\hline 2.94 & 0.11850 & 0.7552 & 0.7413 \\
\hline
\end{tabular}

\begin{tabular}{|c|c|c|c|c|}
\hline \multirow{2}{*}{$\begin{array}{l}\text { Energies of } \\
\text { Irradiation }\end{array}$} & \multirow{2}{*}{$\begin{array}{c}\text { Ion fluences } \\
\left(1 \times 10^{16} \mathrm{~cm}^{-2}\right)\end{array}$} & \multicolumn{3}{|c|}{$\begin{array}{c}\text { The sputtering yields } \\
\text { (Number of sputtered atoms per incident ion) }\end{array}$} \\
\hline & & W atom & O atom & Si atom \\
\hline \multirow{27}{*}{$50 \mathrm{keV}$} & 0.02 & 0.1369 & 1.283 & 1.147 \\
\hline & 0.06 & 0.1462 & 1.288 & 1.105 \\
\hline & 0.10 & 0.1407 & 1.199 & 1.035 \\
\hline & 0.14 & 0.1486 & 1.187 & 1.003 \\
\hline & 0.18 & 0.1524 & 1.156 & 1.009 \\
\hline & 0.22 & 0.1539 & 1.172 & 1.043 \\
\hline & 0.26 & 0.1629 & 1.198 & 1.041 \\
\hline & 0.30 & 0.1578 & 1.231 & 0.979 \\
\hline & 0.34 & 0.1615 & 1.204 & 0.999 \\
\hline & 0.38 & 0.1707 & 1.289 & 1.027 \\
\hline & 0.42 & 0.1575 & 1.217 & 1.022 \\
\hline & 0.46 & 0.1553 & 1.235 & 1.021 \\
\hline & 0.50 & 0.1638 & 1.312 & 1.102 \\
\hline & 0.54 & 0.1684 & 1.282 & 1.080 \\
\hline & 0.58 & 0.1869 & 1.369 & 1.075 \\
\hline & 0.62 & 0.1597 & 1.284 & 1.038 \\
\hline & 0.66 & 0.1800 & 1.355 & 1.151 \\
\hline & 0.70 & 0.1688 & 1.299 & 1.046 \\
\hline & 0.74 & 0.1792 & 1.359 & 1.115 \\
\hline & 0.78 & 0.1709 & 1.292 & 1.048 \\
\hline & 0.82 & 0.1730 & 1.320 & 1.103 \\
\hline & 0.86 & 0.1602 & 1.238 & 1.091 \\
\hline & 0.90 & 0.1729 & 1.283 & 1.111 \\
\hline & 0.94 & 0.1695 & 1.290 & 1.087 \\
\hline & 0.98 & 0.1659 & 1.281 & 1.099 \\
\hline & 1.02 & 0.1791 & 1.304 & 1.130 \\
\hline & 1.06 & 0.1685 & 1.251 & 1.076 \\
\hline
\end{tabular}




\begin{tabular}{|c|c|c|c|c|}
\hline \multirow{48}{*}{$50 \mathrm{keV}$} & 1.10 & 0.1642 & 1.260 & 1.074 \\
\hline & 1.14 & 0.1840 & 1.342 & 1.131 \\
\hline & 1.18 & 0.1826 & 1.317 & 1.095 \\
\hline & 1.22 & 0.1722 & 1.280 & 1.157 \\
\hline & 1.26 & 0.1666 & 1.293 & 1.136 \\
\hline & 1.30 & 0.1781 & 1.323 & 1.162 \\
\hline & 1.34 & 0.1760 & 1.316 & 1.133 \\
\hline & 1.38 & 0.1825 & 1.278 & 1.123 \\
\hline & 1.42 & 0.1776 & 1.268 & 1.129 \\
\hline & 1.46 & 0.1710 & 1.266 & 1.150 \\
\hline & 1.50 & 0.1761 & 1.315 & 1.158 \\
\hline & 1.54 & 0.1835 & 1.287 & 1.186 \\
\hline & 1.58 & 0.1734 & 1.271 & 1.159 \\
\hline & 1.62 & 0.1758 & 1.239 & 1.150 \\
\hline & 1.66 & 0.1669 & 1.268 & 1.167 \\
\hline & 1.70 & 0.1902 & 1.286 & 1.180 \\
\hline & 1.74 & 0.1771 & 1.237 & 1.225 \\
\hline & 1.78 & 0.1753 & 1.268 & 1.166 \\
\hline & 1.82 & 0.1870 & 1.267 & 1.179 \\
\hline & 1.86 & 0.1799 & 1.244 & 1.164 \\
\hline & 1.90 & 0.1870 & 1.295 & 1.254 \\
\hline & 1.94 & 0.1680 & 1.183 & 1.104 \\
\hline & 1.98 & 0.1825 & 1.204 & 1.203 \\
\hline & 2.02 & 0.1799 & 1.206 & 1.185 \\
\hline & 2.06 & 0.1945 & 1.271 & 1.214 \\
\hline & 2.10 & 0.1775 & 1.223 & 1.216 \\
\hline & 2.14 & 0.1850 & 1.251 & 1.171 \\
\hline & 2.18 & 0.1960 & 1.303 & 1.204 \\
\hline & 2.22 & 0.1949 & 1.287 & 1.214 \\
\hline & 2.26 & 0.1950 & 1.205 & 1.199 \\
\hline & 2.30 & 0.1840 & 1.186 & 1.196 \\
\hline & 2.34 & 0.1855 & 1.214 & 1.183 \\
\hline & 2.38 & 0.1877 & 1.202 & 1.160 \\
\hline & 2.42 & 0.1913 & 1.240 & 1.234 \\
\hline & 2.46 & 0.1955 & 1.225 & 1.180 \\
\hline & 2.50 & 0.1896 & 1.232 & 1.235 \\
\hline & 2.54 & 0.1936 & 1.259 & 1.230 \\
\hline & 2.58 & 0.2056 & 1.256 & 1.265 \\
\hline & 2.62 & 0.1824 & 1.147 & 1.239 \\
\hline & 2.66 & 0.2049 & 1.266 & 1.214 \\
\hline & 2.70 & 0.1947 & 1.187 & 1.233 \\
\hline & 2.74 & 0.1948 & 1.233 & 1.252 \\
\hline & 2.78 & 0.1983 & 1.219 & 1.256 \\
\hline & 2.82 & 0.1982 & 1.199 & 1.239 \\
\hline & 2.86 & 0.1904 & 1.166 & 1.207 \\
\hline & 2.90 & 0.1821 & 1.165 & 1.255 \\
\hline & 2.94 & 0.2047 & 1.211 & 1.256 \\
\hline & 2.98 & 0.1960 & 1.153 & 1.272 \\
\hline
\end{tabular}




\begin{tabular}{|c|c|c|c|c|}
\hline \multirow{2}{*}{$\begin{array}{l}\text { Energies of } \\
\text { Irradiation }\end{array}$} & \multirow{2}{*}{$\begin{array}{c}\text { Ion fluences } \\
\left(1 \times 10^{16} \mathrm{~cm}^{-2}\right)\end{array}$} & \multicolumn{3}{|c|}{$\begin{array}{l}\text { The sputtering yields } \\
\text { (Number of sputtered atoms per incident ion) }\end{array}$} \\
\hline & & W atom & O atom & Si atom \\
\hline \multirow{46}{*}{$200 \mathrm{keV}$} & 0.02 & 0.0998 & 0.8384 & 1.0470 \\
\hline & 0.06 & 0.0958 & 0.8191 & 1.0240 \\
\hline & 0.10 & 0.1000 & 0.8101 & 0.9955 \\
\hline & 0.14 & 0.0955 & 0.7789 & 0.9852 \\
\hline & 0.18 & 0.1068 & 0.8332 & 1.0340 \\
\hline & 0.22 & 0.0969 & 0.7913 & 0.9706 \\
\hline & 0.26 & 0.1067 & 0.8345 & 0.9509 \\
\hline & 0.30 & 0.1002 & 0.7794 & 0.9395 \\
\hline & 0.34 & 0.1063 & 0.8149 & 0.9445 \\
\hline & 0.38 & 0.1073 & 0.8328 & 0.9470 \\
\hline & 0.42 & 0.1077 & 0.8248 & 0.9538 \\
\hline & 0.46 & 0.1106 & 0.8209 & 0.9238 \\
\hline & 0.50 & 0.1155 & 0.8425 & 0.9613 \\
\hline & 0.54 & 0.1113 & 0.8407 & 0.9347 \\
\hline & 0.58 & 0.1143 & 0.8583 & 0.9400 \\
\hline & 0.62 & 0.1155 & 0.8495 & 0.9507 \\
\hline & 0.66 & 0.1114 & 0.8293 & 0.9413 \\
\hline & 0.70 & 0.1147 & 0.8771 & 0.9408 \\
\hline & 0.74 & 0.1119 & 0.8330 & 0.9498 \\
\hline & 0.78 & 0.1135 & 0.8753 & 0.9497 \\
\hline & 0.82 & 0.1149 & 0.8503 & 0.9212 \\
\hline & 0.86 & 0.1246 & 0.9091 & 0.9797 \\
\hline & 0.90 & 0.1194 & 0.8855 & 0.9680 \\
\hline & 0.94 & 0.1136 & 0.8588 & 0.9402 \\
\hline & 0.98 & 0.1216 & 0.8961 & 0.9704 \\
\hline & 1.02 & 0.1211 & 0.8885 & 0.9568 \\
\hline & 1.06 & 0.1225 & 0.8992 & 0.9995 \\
\hline & 1.10 & 0.1202 & 0.8660 & 0.9696 \\
\hline & 1.14 & 0.1123 & 0.8498 & 0.9475 \\
\hline & 1.18 & 0.1238 & 0.9181 & 0.9739 \\
\hline & 1.22 & 0.1180 & 0.8764 & 0.9472 \\
\hline & 1.26 & 0.1199 & 0.8748 & 0.9534 \\
\hline & 1.30 & 0.1342 & 0.9381 & 0.9845 \\
\hline & 1.34 & 0.1220 & 0.8841 & 0.9460 \\
\hline & 1.38 & 0.1218 & 0.8887 & 0.9820 \\
\hline & 1.42 & 0.1295 & 0.9130 & 0.9997 \\
\hline & 1.46 & 0.1268 & 0.8893 & 0.9769 \\
\hline & 1.50 & 0.1168 & 0.8339 & 0.9375 \\
\hline & 1.54 & 0.1194 & 0.8819 & 0.9583 \\
\hline & 1.58 & 0.1309 & 0.9310 & 1.0200 \\
\hline & 1.62 & 0.1299 & 0.9047 & 0.9735 \\
\hline & 1.66 & 0.1178 & 0.8465 & 0.9584 \\
\hline & 1.70 & 0.1289 & 0.8907 & 0.9657 \\
\hline & 1.74 & 0.1294 & 0.8731 & 0.9657 \\
\hline & 1.78 & 0.1219 & 0.8679 & 0.9886 \\
\hline & 1.82 & 0.1266 & 0.8819 & 1.0030 \\
\hline
\end{tabular}




\begin{tabular}{|c|c|c|c|c|}
\hline \multirow{29}{*}{$200 \mathrm{keV}$} & 1.86 & 0.1240 & 0.8742 & 0.9848 \\
\hline & 1.90 & 0.1297 & 0.8799 & 0.9753 \\
\hline & 1.94 & 0.1335 & 0.8862 & 1.0010 \\
\hline & 1.98 & 0.1231 & 0.8867 & 0.9527 \\
\hline & 2.02 & 0.1273 & 0.8650 & 0.9901 \\
\hline & 2.06 & 0.1300 & 0.9015 & 1.0010 \\
\hline & 2.10 & 0.1205 & 0.8254 & 0.9642 \\
\hline & 2.14 & 0.1244 & 0.8550 & 0.9965 \\
\hline & 2.18 & 0.1248 & 0.8700 & 0.9751 \\
\hline & 2.22 & 0.1240 & 0.8515 & 0.9716 \\
\hline & 2.26 & 0.1239 & 0.8630 & 0.9886 \\
\hline & 2.30 & 0.1244 & 0.8471 & 0.9801 \\
\hline & 2.34 & 0.1271 & 0.8474 & 0.9832 \\
\hline & 2.38 & 0.1280 & 0.8590 & 0.9903 \\
\hline & 2.42 & 0.1289 & 0.8359 & 0.9998 \\
\hline & 2.46 & 0.1298 & 0.8566 & 1.0040 \\
\hline & 2.50 & 0.1273 & 0.8475 & 0.9680 \\
\hline & 2.54 & 0.1279 & 0.8512 & 0.9860 \\
\hline & 2.58 & 0.1362 & 0.8857 & 0.9996 \\
\hline & 2.62 & 0.1302 & 0.8243 & 0.9628 \\
\hline & 2.66 & 0.1282 & 0.8510 & 0.9907 \\
\hline & 2.70 & 0.1212 & 0.8235 & 0.9844 \\
\hline & 2.74 & 0.1275 & 0.8205 & 0.9616 \\
\hline & 2.78 & 0.1340 & 0.8420 & 0.9822 \\
\hline & 2.82 & 0.1259 & 0.7909 & 0.9609 \\
\hline & 2.86 & 0.1337 & 0.8360 & 0.9926 \\
\hline & 2.90 & 0.1307 & 0.8310 & 1.0020 \\
\hline & 2.94 & 0.1281 & 0.8493 & 0.9816 \\
\hline & 2.98 & 0.1310 & 0.8327 & 0.9916 \\
\hline
\end{tabular}

Video S1: This video shows role of bending moment while cutting silicon substrate.

Video S2: This video shows the superhydrophilic nature of the $\mathrm{WO}_{3}$ nanorod film surface 